\title{
A bronchoscopy-associated pseudo-outbreak of Mycobacterium mucogenicum traced to use of contaminated ice used for bronchoalveolar lavage
}

\author{
Judie Bringhurst MSN, RN, $\mathrm{CIC}^{1}$, David J. Weber MD, MPH ${ }^{1,2}$, Melissa B. Miller PhD ${ }^{3,4}$, Melissa C. Jones $\mathrm{BS}^{3}$, \\ M. Patricia Rivera MD ${ }^{5}$, Jason Akulian MD, MPH ${ }^{5}$, William A. Rutala PhD, MPH ${ }^{2}$ and Emily E. Sickbert-Bennett PhD, MS ${ }^{1,2}$ \\ ${ }^{1}$ Hospital Epidemiology, University of North Carolina Hospitals, Chapel Hill, North Carolina, ${ }^{2}$ Division of Infectious Diseases, UNC School of Medicine, Chapel Hill, \\ North Carolina, ${ }^{3}$ Clinical Microbiology Laboratory, University of North Carolina Hospitals, Chapel Hill, North Carolina, ${ }^{4}$ Department of Pathology and Laboratory \\ Medicine, UNC School of Medicine, Chapel Hill, North Carolina and ${ }^{5}$ Division of Pulmonary and Critical Care Medicine, UNC School of Medicine, Chapel Hill, North \\ Carolina
}

\begin{abstract}
Clonal Mycobacterium mucogenicum isolates (determined by molecular typing) were recovered from 19 bronchoscopic specimens from 15 patients. None of these patients had evidence of mycobacterial infection. Laboratory culture materials and bronchoscopes were negative for Mycobacteria. This pseudo-outbreak was caused by contaminated ice used to provide bronchoscopic lavage. Control was achieved by transitioning to sterile ice.
\end{abstract}

(Received 23 July 2019; accepted 11 October 2019; electronically published 15 November 2019)

Flexible bronchoscopy is a commonly used procedure for the diagnosis and treatment of pulmonary disorders. Importantly, $>50$ outbreaks and pseudo-outbreaks have been associated with bronchoscopy. ${ }^{1-3}$ Outbreaks and pseudo-outbreaks have most commonly involved Pseudomonas aeruginosa, Mycobacterium tuberculosis, and nontuberculous mycobacteria (NTM).

The use of contaminated ice has led to multiple outbreaks in healthcare settings. ${ }^{4,5}$ Common pathogens linked to the use of contaminated ice have included Legionella spp, gram-negative bacilli (eg, Pseudomonas, Enterobacter), and NTM (eg, M.chelonae, M.porcinum) ${ }^{4,5}$ However, pseudo-outbreaks of Mycobacterium mucogenicum caused by ice used during bronchoscopy have not been described.

Here, we report a pseudo-outbreak of bronchoscopy-associated M. mucogenicum traced to contaminated ice used for bronchoalveolar lavage (BAL) involving 15 patients, 11 bronchoscopes, and 5 physicians or practitioners at UNC Hospitals. We describe the investigation of this pseudo-outbreak, and we demonstrate the clonality of M. mucogenicum and the link to contaminated ice by molecular analysis. We further discuss the methods of preventing ice-associated outbreaks and pseudo-outbreaks.

\section{Outbreak investigation}

In February 2017, the infection prevention team received notification from the microbiology laboratory that 9 bronchoscopy

\footnotetext{
Author for correspondence: Judie Bringhurst, Email: judie.bringhurst@unchealth. unc.edu

Cite this article: Bringhurst J, et al. (2020). A bronchoscopy-associated pseudooutbreak of Mycobacterium mucogenicum traced to use of contaminated ice used for bronchoalveolar lavage. Infection Control \& Hospital Epidemiology, 41: 124-126, https://doi.org/10.1017/ice.2019.317
}

specimens from 8 patients had yielded M. mucogenicum. All had been isolated from fungal cultures of bronchoscopy-obtained specimens. Ultimately, 19 respiratory samples from 15 patients yielded cultures positive for M. mucogenicum (see Fig. 1 for the epidemic curve). Upon receipt of the initial report of $M$. mucogenicum, the infection prevention team began an outbreak investigation that focused on whether this finding represented an outbreak or a pseudo-outbreak and on the source of the M. mucogenicum. A retrospective review indicated that the prevalence of $M$. mucogenicum from bronchoscopy-obtained specimens consisted of 1 specimen from 2015 and 1 specimen from 2016.

A review of the medical records of the involved patients revealed 7 female and 8 male patients ranging in age from 27 to 79 years. There were multiple indications for bronchoscopy with 12 patients having a prebronchoscopy diagnosis of cancer. To assess the clinical significance of the M. mucogenicum isolates, patient charts were reviewed by board-certified pulmonologists for signs and/or symptoms of NTM infection. No patient had a clinical illness compatible with NTM infection. Patients were notified of their exposure to M. mucogenicum via personal telephone call from their pulmonologist and were assessed for infection. No patients were reported to have a clinical illness compatible with NTM infection. Based on this evaluation, the M. mucogenicum isolates were considered a pseudo-outbreak.

An evaluation of culture methods by the microbiology laboratory eliminated contaminated culture plates or re-usable pipettes as a cause of this pseudo-outbreak. The bronchoscopes and automated endoscope reprocessor (AER) used for high-level disinfection of the bronchoscopes were also investigated. Bronchoscope reprocessing records, including paper logs for minimum effective concentration documentation, AER records, filter change logs, and 
Table 1. Results of Pulsed-Field Gel Electrophoresis (PFGE) of 9 Isolates From Patients and 1 Isolate From an Ice Machine Using 2 Restriction Enzymes

\begin{tabular}{llccc}
\hline Isolate No. & Source & Collection Date & Xbal & Asel \\
\hline 1 & Patient & $01 / 26 / 2017$ & A1 & B \\
\hline 2 & Ice machine & $02 / 20 / 2017$ & A1 & B \\
\hline 3 & Patient & $02 / 15 / 2017$ & A2 & B \\
\hline 4 & Patient & $01 / 30 / 2017$ & A1 & B \\
\hline 5 & Patient & $02 / 02 / 2017$ & A2 & B \\
\hline 6 & Patient & $02 / 09 / 2017$ & A1 & B \\
\hline 7 & Patient & $2 / 16 / 2017$ & A1 & B \\
\hline 8 & Patient & $02 / 08 / 2017$ & A1 & B \\
\hline 9 & Patient & $01 / 19 / 2017$ & A1 & B \\
\hline
\end{tabular}

Note. Asel, all the patient isolates and the environmental isolate were indistinguishable from each other. Xbal, all the isolates were indistinguishable from each other, except isolates 3 and 5 , which differed by just 1 band from the majority group and thus were considered closely related or clonal. Isolates 3 and 5 were indistinguishable from each other by Xba I.

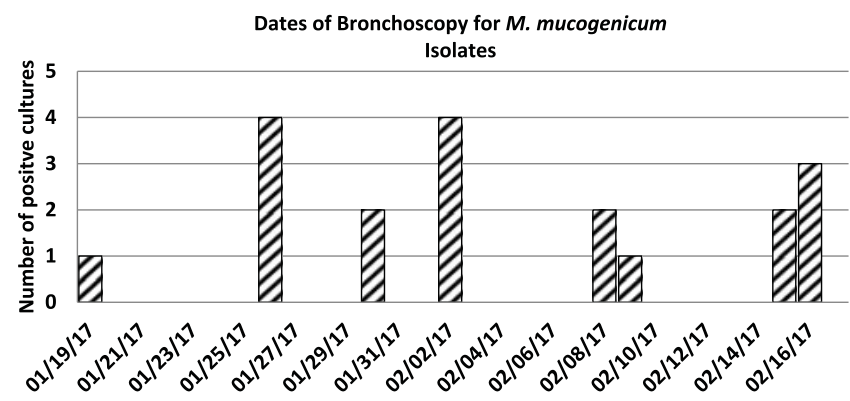

Fig. 1. Outbreak curve displaying dates of bronchoscopy yielding positive respiratory cultures for Mycobacterium mucogenicum. Cases of Mycobacterium mucogenicum in brochoscopically obtained respiratory samples; hatched lines are cases linked to current outbreak.

periodic maintenance logs, were inspected. No irregularities were found. Cultures of glutaraldehyde from the reservoirs of the AER were negative. We cultured 8 of the 11 implicated bronchoscopes involved in the 15 procedures, and all were negative.

The bronchoscopy methods, procedures, and locations were evaluated. All 15 procedures were performed in 3 rooms within the operating room suite. During our evaluation, it was discovered that nonsterile ice from 2 ice machines was being mixed with sterile saline to form a slurry used for bronchoalveolar lavage to reduce the risk of bleeding. The use of nonsterile ice ceased. Cultures of both ice machines were positive for $M$. mucogenicum.

Overall, 8 isolates of M. mycogenicum from unique patients and 1 isolate from an ice machine were evaluated by pulsed-field gel electrophoresis (PFGE) using the restriction enzymes Xbal and Asel. All 9 isolates were clonal, as demonstrated by PFGE (Table 1).

Multiple control measures were implemented because of this pseudo-outbreak. Although both ice machines had been maintained according to hospital policy, both machines were taken out of service and underwent standard cleaning and disinfection. A meeting was convened of all stakeholders during which it was revealed that staff may have believed that since bronchoscopy was not a sterile procedure it was acceptable to use nonsterile ice for lavage. Staff were educated on the risks of instilling nonsterile material (eg, ice) into body cavities. Sterile ice was made available for future lavage.

\section{Discussion}

This pseudo-outbreak highlights several important issues regarding outbreaks, including the frequency of bronchoscope-associated outbreaks, the importance of NTM as a source of outbreaks, and the role of nonsterile ice and water in outbreaks. More than 50 outbreaks and pseudo-outbreaks have been linked to bronchoscopy, most commonly due to inadequate cleaning and/or disinfection. ${ }^{1-3}$ Pseudo-outbreaks have also been linked to use of contaminated water including a pseudo-outbreak of Legionella linked to use of nonsterile ice for bronchoalveolar lavage. ${ }^{4,5}$

Mycobacteria have been well studied, and the genus currently contains $>100$ species. Pseudo-outbreaks of NTM have been well described, ${ }^{6}$ including pseudo-outbreaks linked to bronchoscopy. ${ }^{2,3,6}$ Most pseudo-outbreaks of rapidly growing mycobacteria (RGM) in health care have been epidemiologically linked to various water sources, including ice. Members of the Mycobacterium mucogenicum group (M. mucogenicum, Mycobacterium aubagnense, and Mycobacterium phocaicum), a subgroup of RGM, are ubiquitous; they are present in public water systems and hospital water systems and have recently even been found in chili sauces sold by street vendors in Mexico. ${ }^{7}$ Their persistence may be linked to their ability to tolerate disinfectants, chlorination, and extreme temperatures. ${ }^{7,8}$ Mycobaterium mucogenicum is infrequently recovered from true infections; however, exposure to tap water seems to be the most frequent risk factor for healthcare-associated infections. ${ }^{8,9}$

To distinguish between an outbreak and a pseudo-outbreak, an outbreak investigation should always include an assessment of whether the positive cultures or diagnostic tests led to infections. ${ }^{10}$ Uncovering pseudo-outbreaks is important to prevent unnecessary diagnostic tests and/or treatment of patients. As noted, multiple pseudo-outbreaks have been linked to bronchoscopy. ${ }^{1-3}$ Our evaluation included a review of the culture methods used in our microbiology laboratory that failed to identify a laboratory source for NTM contamination. Water sources (including ice) are well described as sources of healthcare-associated outbreaks and pseudo-outbreaks. ${ }^{4,5}$ Contaminated ice has been linked to outbreaks or pseudo-outbreaks due to Legionella, gram-negative bacilli, and mycobacteria. ${ }^{5}$ In our pseudo-outbreak, the M. mucogenicum isolated from our patients and the operating rooms ice machines were demonstrated to be clonal by PGFE.

The goal of an outbreak investigation is to prevent future outbreaks, and we accomplished this by mandating that only sterile ice be used for bronchoscopic lavage. The key findings of our investigation are to consider pseudo-outbreaks in evaluating a cluster of positive cultures linked to a procedure and to assure that only sterile ice is used for instillation of fluids into internal body cavities.

Acknowledgments. We thank the UNC Medical Center Clinical Microbiology Laboratory for their role in the identification of a potential outbreak and for investigating potential sources in the laboratory. We also thank Dr. Richard Wallace for performing the pulsed-field gel analysis and Ms. Lauren DiBiase for providing infection prevention surveillance data.

Financial support. No financial support was provided relevant to this article.

Conflicts of interest. All authors report no conflicts of interest relevant to this article.

\section{References}

1. Seoane-Vazquez E, Rodriguez-Monguio R, Visaria J, Carlson A. Exogenous endoscopy-related infections, pseudoinfections, and toxic reactions: clinical and economic burden. Curr Med Res Opin 2006;22:2007-2021. 
2. Weber DJ, Rutala WA. Lessons learned from outbreaks and pseudooutbreaks associated with bronchoscopy. Infect Control Hosp Epidemiol 2012;33:230-234.

3. Kovaleva J, Peters FT, van der Mei HC, Degener JE. Transmission of infection by flexible gastrointestinal endoscopy and bronchoscopy. Clin Microbiol Rev 2013;26:231-254.

4. Decker BK, Palmore TN. The role of water in healthcare-associated infections. Curr Opin Infect Dis 2013;26:345-351.

5. Kanamori H, Weber DJ, Rutala WA. Healthcare outbreaks associated with a water reservoir and infection prevention strategies. Clin Infect Dis 2016;62: $1423-1435$.
6. Wallace RJ Jr, Brown BA, Griffith DE. Nosocomial outbreaks/pseudo-outbreaks caused by nontuberculous mycobacteria. Annu Rev Microbiol 1998;52:453-490.

7. Adékambi T. Mycobacterium mucogenicum group infections: a review. Clin Microbiol Infect 2009;15:911-918.

8. Brown-Elliott BA, Philley JV. Rapidly growing mycobacteria. Microbiol Spectr 2017;5(1). doi: 10.1128/microbiolspec.

9. Rodriguez-Coste MA, Chirca I, Steed LL, Salgado CD. Epidemiology of rapidly growing mycobacteria bloodstream infections. Am J Med Sci 2016;351:253-258.

10. Cunha CB, Cunha BA. Pseudoinfections and pseudo-outbreaks. In: C. Glen Mayhall, ed. Hospital Epidemiology and Infection Control, 4th edition. Philadelphia: Lippincott Williams \& Wilkins; 2012:142-152. 